\title{
Retinal Nerve Fibre Layer and Ophthalmic Artery Blood Parameters in Pseudoexfoliation Syndrome and Pseudoexfoliative Glaucoma
}

Hacı Koç ( $\square$ hacikoc@gmail.com )

Pivate Adatıp Hospital https://orcid.org/0000-0002-5446-8456

\section{Faruk Kaya}

Istanbul Medipol University Faculty of Medicine: Istanbul Medipol Universitesi Tip Fakultesi

Ümit İpeksoy

Private Adatıp Hospital, Sakarya

\section{Research Article}

Keywords: Retinal Nerve Fibre, Ophthalmic Artery Blood, Pseudoexfoliation Syndrome, Pseudoexfoliative Glaucoma

Posted Date: July 15th, 2021

DOl: https://doi.org/10.21203/rs.3.rs-704975/v1

License: (a) (1) This work is licensed under a Creative Commons Attribution 4.0 International License. Read Full License 


\section{Abstract}

Purpose

This study investigates the relationship between ophthalmic artery blood flow parameters and retinal nerve fiber layer (RNFL) thickness in eyes with pseudoexfoliation.

\section{Methods}

Three comparisons were made in this study. In the first, eyes without glaucoma with pseudoexfoliation in any of the eyes were compared with the control group. In the second, eyes with pseudoexfoliation and without glaucoma were compared with those with pseudoexfoliation and glaucoma. In the third, cases with glaucoma and pseudoexfoliation were compared with the control group. Ophthalmic artery colour Doppler imaging measurements were performed in all patients. Also, peripapillary RNFL analysis was performed.

Results

A statistically significant difference was found between the total RNFL measurements according to group ( $p=$ $0.012 ; p<0.05)$. From the paired comparisons, the total RNFL measurements from the control group were higher than the group without glaucoma $(p=0.010)$. No statistically significant difference was found between the total RNFL measurements of the other groups $(p>0.05)$. In the pseudoexfoliative glaucoma (PXG) group, there was a statistically significant negative correlation between total RNLF and Peak systolic velocity (PSV) $(r=-0.743 ; p<$ 0.01). There was also a statistically significant negative correlation between total RNLF and End diastolic velocity $(E D V)(r=-0.691 ; p<0.01)$. There was no significant relationship between total RNLF and resistive index $(R I)$ measurements $(p>0.05)$.

\section{Conclusion}

Pseudoexfoliation syndrome(PXS) caused a decrease in the PSV and EDV values of ophthalmic artery blood flow parameters. Again, PXS caused a decrease in total RNFL, RNFL S, RNFL I and RNFL T thickness values.

\section{Introduction}

PXS is an age-related syndrome. It is characterized by the production and progressive storage of small, white deposits of fibrillar extracellular psedoexfoliative material in ocular tissues [1]. Pseudoexfoliative material can be observed in ocular and extraocular tissues such as lens capsule, ciliary body, anterior and posterior surface of the iris, pupillary rim, trabecular meshwork, conjunctival stroma, corneal endothelium, extraocular muscles, optic nerve meninges, orbital connective tissue, skin, heart, kidney, bladder, lungs, liver, cerebral meninges, adventitia of the aorta and cerebral artery [2-4].

Blood flow parameters can be altered by the storage of pseudoexfoliative material in the walls of iris veins, posterior ciliary arteries, vortex veins and central retinal veins. This can lead to increased permeability, narrowing and eventually obstruction [1]. In a study conducted on patients who had at least one transient ischemic attack, high resistivity indices (RIs) for ophthalmic arteries included pseudoexfoliative material at a high frequency [5].

Colour Doppler imaging (CDI) is a reliable, non-invasive, reproducible method of evaluating orbital blood flow velocities. It has been increasingly used to assess pathologies such as vascular occlusion, tumors, glaucoma and 
caroticocavernous fistula that affect orbital haemodynamics [6-9].

Visual field evaluation and optical coherence tomography (OCT) can provide functional data and information on the anatomical structure of the retinal nerve fibre layer (RNFL) [10]. Early structural changes in the thickness of the RNFL in patients with PXS have been shown primarily by optical coherence tomography studies [11-13]. Some studies suggest that OCT is more sensitive and practical than other tests (e.g., visual field scanning) for detecting glaucomatous damage in the optic nerve head [14-16].

In this study, we aimed to evaluate the relationship between ophthalmic artery blood flow parameters and RNFL thickness in eyes with pseudoexfoliation.

\section{Materials And Methods}

This prospective study was conducted at the Adatip Hospital in accordance with the principles of the Declaration of Helsinki. The study was reviewed and approved by the Private Istanbul Medipol University Ethics Committee (10840098-604.01.01-E.12134/10.03.2020). All patients provided written informed consent.

All patients underwent routine ophthalmologic examination. The best corrected visual acuities were obtained, slitlamp biomicroscopic examinations were performed in the miotic and mydriatic state, intraocular pressure measurements were taken, angle examinations were performed with a Goldmann three-mirror lens, fundus examinations were performed with a 90 diopter lens, and the RNFL with an OCT device. RNFL data were analyzed. Total retinal thickness and four quadrant nerve and fiber layer values were compared. Detailed medical histories from all patients were obtained. Patients with cardiovascular disease other than systemic hypertension and patients with diabetic retinopathy were excluded from the study. Patients with systemic hypertension with their systemic blood pressure under control were included in the study.

Patients with pseudoexfoliative material on the pupillary rim or anterior capsule surface after mydriasis were included in the group of eyes with pseudoexfoliation. Patients with intraocular pressure $<21 \mathrm{mmHg}$, diurnal IOP fluctuation within normal limits ( $\leq 5 \mathrm{mmHg}$ ), no glaucomatous optic nerve changes, and normal visual field scans were included in the group of patients with PXS. Patients with pseudoexfoliative material detected and glaucomatous optic nerve changes (vertical linear cup to disc ratio $>0.6$, notching of the neuroretinal rim) and pathological visual field scans were included in the group of patients with PXG. We confirmed that there was no pseudoexfoliation in the patients included in the control group while their pupils were dilated. Three comparisons were made in the study. In the first, the control group was compared with in eyes without glaucoma and pseudoexfoliation. In the second, eyes with pseudoexfoliation with and without glaucoma were compared. In the third, those with glaucoma and the control group were compared.

Also, ophthalmic artery Color Doppler Imaging (CDI) was performed in all patients by the same experienced specialist (U.I.) using Doppler sonography (Affinity 70G Philips, probe 5-12 MHz). The eyelids of the patients were closed using acoustic gel, with pressure applied to the eyes at 7.5-MHz. CDI measurements were made with a linear transducer. The patients were examined in the supine position and asked to close their eyes and look straight ahead. Ophthalmic artery blood parameters were obtained approximately $25 \mathrm{~mm}$ behind the globe, where the artery crossed the optic nerve. 
Peripapillary RNFL analysis was performed with a spectral-domain OCT (3D OCT-1 Maestro, Topcon, Tokyo, Japan) device. Each papilla was analysed over four quadrants: temporal quadrant thickness $\left(316-45^{\circ}\right)$, superior quadrant thickness $\left(46-135^{\circ}\right)$, nasal quadrant thickness $\left(136-225^{\circ}\right)$, and inferior quadrant thickness $\left(226-315^{\circ}\right)$. In addition, each eye included in the study was evaluated in terms of total RNFL thickness.

Statistical Analysis

The NCSS (Number Cruncher Statistical System) 2007 (Kaysville, UT, USA) program was used for statistical analysis. Descriptive statistical methods (mean, standard deviation, frequency, ratio, minimum, maximum) were used to evaluate the study data. The suitability of the quantitative data to normal distribution was tested by the Kolmogorov-Smirnov, Shapiro-Wilk's test and graphical evaluations. One-way ANOVA was used to compare three or more normally distributed groups, and the Bonferroni test was used for paired comparisons. Significance was assigned at the $p<0.05$ level.

\section{Results}

The study was carried out at the Private Adatıp Hospital Eye Clinic between March 2020 and March 2021 with 115 eye measurements from a total of 95 cases. The right eye of $27.5 \%(n=14)$ of the cases $(n=51)$, the left eye of $33.3 \%(n=17)$ and both eyes of $39.2 \%(n=20)$ of the cases were included in the study. Glaucoma was not detected in $74.6 \%(n=53)$ of the115 eyes included in the study and was found in $25.4 \%(n=18)$ (Table 1$)$.

A statistically significant difference was found between the total RNFL measurements according to the groups ( $p$ $=0.012 ; p<0.05)$. From the paired comparisons, the mean total RNFL measurements from the control group were higher than from the group without glaucoma $(p=0.010)$. No statistically significant difference was found between the total RNFL measurements of the other groups $(p>0.05)$ (Table 2, Figure 1$)$.

A statistically significant difference was found for the PSV measurements according to group $(p=0.001 ; p<$ 0.01). From the paired comparison, PSV measurements of cases with and without glaucoma were lower than the control group ( $p=0.007 ; p=0.001$, respectively). There was no statistically significant difference between the PSV measurements from the groups with and without glaucoma ( $p>0.05)$ (Figure 2).

A statistically significant difference was found for the EDV measurements according to the groups $(p=0.001 ; p<$ 0.01). From the paired comparisons, the EDV measurements of patients with and without glaucoma were lower than the control group ( $p=0.004 ; p=0.001$, respectively). There was no statistically significant difference between the EDV measurements of the groups with and without glaucoma ( $p>0.05)$ (Figure 3 ).

There was no statistically significant difference in the RI measurements according to group $(p>0.05)$.

A statistically significant difference was found for the total RNFL measurements according to the groups $(p=$ 0.012; $p$ 0.05). From the paired comparisons, the total RNFL measurements of the cases control group were higher than the without glaucoma $(p=0.010)$. No statistically significant difference was found between the total RNFL measurements of the other groups ( $p>0.05)$ (Table 3$)$.

A statistically significant difference was found for the RNFL $S$ measurements according to the groups $(p=0.001$; $p<0.01)$. From the paired comparisons, the RNFL $S$ measurements of patients with and without glaucoma were 
lower than the control group ( $p=0.001 ; p=0.004$, respectively). RNFL $S$ measurements of cases with glaucoma were also lower than cases without glaucoma $(p=0.010 ; p<0.05)$ (Figure 4).

A statistically significant difference was found for the RNFL I measurements according to the groups $(p=0.001 ; p$ $<0.01$ ). From the paired comparisons, the RNFL I measurements of the patients with glaucoma were lower than those without glaucoma and the control group $(p=0.001 ; p=0.001$, respectively). There was no statistically significant difference between the RNFL I measurements of the group without glaucoma and the control group ( $p$ $>0.05)$.

A statistically significant difference was found for the RNFL T measurements according to the groups $(p=0.001$; $p<0.01)$. From the paired comparisons, the RNFL T measurements of the cases with and without glaucoma were lower than the control group ( $p=0.001 ; p=0.028$, respectively). There was no statistically significant difference for the RNFL T measurements of the groups with and without glaucoma ( $p$ > 0.05) (Figure 5).

There was no statistically significant difference for the RNFL N measurements according to the groups $(p>0.05)$.

A weak negative correlation was found between total RNLF and PSV and EDV measurements in the PEX (+) Glaucoma (-) group, but this was not statistically significant $(p>0.05)$. There was no significant relationship between total RNLF and RI measurements $(p>0.05)$.

In the PEX (+) Glaucoma (+) group, there was a statistically significant negative correlation between total RNLF and PSV $(r=-0.743 ; p<0.01)$. There is also a statistically significant negative correlation between total RNLF and EDV $(r=-0.691 ; p<0.01)$. There was no significant relationship between total RNLF and RI measurements ( $p>$ 0.05).

In the control group, there was a moderately significant positive correlation between total RNLF and PSV in the group ( $r=0.317 ; p<0.05)$. There was also a moderately significant positive correlation between total RNLF and $\operatorname{EDV}(r=0.337 p<0.05)$. There was no significant relationship between total RNLF and RI measurements $(p>$ 0.05).

\section{Discussion}

This study showed that patients with pseudoexfoliation with and without glaucoma had alterations in the hemodynamic parameters of ophthalmic arteries and decreased RNFL thickness.

Ageing is associated with decreased EDV at unchanged PSV values of the ophthalmic artery (OA) [17]. Changes in blood flow velocity lead to increased vascular RI. Increased RI in the analysed group was associated with increased systolic blood pressure (SBP) and decreased RI with increased diastolic blood pressure (DBP). In addition, increased DBP correlates with increased EDV, which leads to decreased RI. Similarly, increased SBP and PSV cause increased RI in the OA [18]. These observations confirm the age-related vascular remodelling theory. Structural changes decrease the diameter of the vessels and increase their rigidity. This remodelling provides the stable velocity of the current in the retrobulbar circulation together with the decreased blood flow volume [19].

Harris et al. found a correlation between OA circulation parameters and sex. They stated that ageing may lead to a $30 \%$ reduction in ophthalmic artery EDV in women and $12 \%$ in men. They reported that ageing was associated with an increase in the RI of OA by $50 \%$ in women and $26 \%$ in men [17]. 
In a study, it was found that hemodynamic parameters in retrobulbar vessels were significantly lower in patients with PXG compared with patients with PXS. Additionally, in the same study, no difference was observed between patients with PXS and the control group in terms of retrobulbar blood flow parameters [1].

In our study, PSV and EDV measurements of the ophthalmic artery were lower in patients with PEX with and without glaucoma compared with the patients in the control group. On the other hand, no significant difference was observed between PEX patients with glaucoma and patients with PEX without glaucoma. Although there was no statistically significant difference between the groups in terms of the RI parameter, the average RI was higher in groups with PEX.

Exfoliative material has been identified in the central retinal artery and short posterior ciliary arteries [3,20], which feed the prelaminar and laminar regions of the superficial nerve fibre layer. These vessels provide vascular support of the optic nerve head [1].

PXS is a systemic disease with marked vascular damage [21,22]. Vascular changes occurring in eyes with pseudoexfoliation are increased vascular permeability, occlusion and disappearance of vessels,

neovascularization and increased RI of the ophthalmic artery. Pseudoexfoliative material accumulates in vascular endothelial cells, smooth muscle cells and pericytes. All of these cell types play regulatory roles in local microcirculation [7].

The level of glaucoma in eyes with pseudoexfoliation is associated with the amount of pseudoexfoliative material deposited in the cribriform region of the optic nerve [23]. In a 10-year study by Puska et al., 38\% of patients with unilateral PEX syndrome progress to bilateral PEX syndrome [24]. Therefore, it is important to follow up on the other eyes of patients with PEX syndrome in one eye.

In many studies, significantly decreased RNFL was found in the inferotemporal quadrant in eyes with PEX syndrome compared with cases in the control group. This finding may be an early sign of retinal nerve fibre damage in the eyes with PEX syndrome, even though the IOP and visual fields are normal [13]. Puska et al. suggest that PEX material may be a risk factor independent of IOP for ganglion cell damage in eyes with PXS and glaucoma [25].

In our study, total RNFL thickness values were observed to be higher in the control group compared with the group of patients with PEX without glaucoma. Total RNFL thickness values of patients with PEX without glaucoma were also detected at higher rates compared with patients with PEX with glaucoma.

When RNFL superior, RNFL inferior and RNFL temporal thickness values were taken as criteria, these values were higher in the control group than the other groups. There was no significant difference between the groups in terms of RNFL nasal. When patients with PEX with and without glaucoma were compared, no significant difference was observed except in RNFL superior thickness. RNFL superior thickness values were lower in patients with glaucoma.

Study limitations

We see the limited number of cases as a factor limiting our study. And the short follow-up period of patients with pseudoexfoliation in only one eye to control the condition of the other eye can be considered as one of the factors limiting our study. 


\section{Conclusions}

Our study shows that there is a decrease in total RNFL thickness values in eyes with pseudoexfoliation. In cases with pseudoexfoliation, PSV and EDV values, which are among the ophthalmic artery parameters, are decreased. Especially in patients with glaucoma and pseudoexfoliation, a decrease in total retinal thickness values, as well as PSV and EDV, is also observed.

\section{Declarations}

Hacı Koc declares that he has no conflict of interest.

Faruk Kaya declares that he has no conflict of interest.

Umit Ipeksoy declares that he has no conflict of interest.

All procedures performed in studies involving human participants were in accordance with the ethical standards of the institutional and/or national research committee and with the 1964 Helsinki declaration and its later amendments or comparable ethical standards.

Informed consent was obtained from all individual participants included in the study.

This article does not contain any studies with animals performed by any of the authors.

\section{References}

1. Martinez A, Sanchez M. (2008). Retrobulbar hemodynamic parameters in pseudoexfoliation syndrome and pseudoexfoliative glaucoma. Graefe's Arch Clin Exp Ophthalmol. 246:1341-1349

2. Kocaturk T, Isikligil I, Uz B, Dayanir V, Dayanır YO (2016). Ophthalmic artery blood flow parameters in pseudoexfoliation glaucoma. Eur J Ophthalmol; 26 (2): 124-127

3. Schlotzer-Schrehardt U, Küchle M, Naumann GO (1991). Electron microscopic identification of pseudoexfoliation material in extrabulbar tissue. Arch Ophthalmol;109:565-70.

4. Dayanir V, Topaloğlu A, Ozsunar Y, Keceli M, Okyay P, Harris A (2009). Orbital blood flow parameters in unilateral pseudoexfoliation syndrome. Int Ophthalmol 29:27-32

5. Repo LP, Suhonen MT, Teräsvirta ME, Koivisto KJ (1995). Color Doppler imaging of the ophthalmic artery blood flow spectra of patients who have had a transient ischemic attack. Correlations with generalized iris transluminance and pseudoexfoliation syndrome. Ophthalmology;102:1199-205.

6. Williamson TH, Harris A (1996). Color Doppler ultrasound imaging of the eye and orbit. Surv Ophthalmol;40:225-67.

7. Yüksel N, Karabaş VL, Arslan A, Demirci A, Çağlar Y (2001). Ocular Hemodynamics in Pseudoexfoliation Syndrome and Pseudoexfoliation Glaucoma. Ophthalmology; 108(6): 1043-49. 
8. Costa VP, Kuzniec S, Molnar LJ, et al (1997). Clinical findings and hemodynamic changes associated with severe occlusive carotid artery disease. Ophthalmology;104:1994-2002.

9. Quaranta L, Harris A, Donato F, et al (1997). Color Doppler imaging of ophthalmic artery blood flow velocity: a study of repeat ability and agreement. Ophthalmology;104:653-8.

10. Demircan S, Yılmaz U, Kucuk E, Ulusoy M.D, Ataş M, Gulhan A, Zararsız G (2017). The Effect of Pseudoexfoliation Syndrome on the Retinal Nerve Fiber Layer and Choroid Thickness. Seminars in Ophthalmology; 32(3):341-347.

11. Yüksel N, Altıntaş Ö, Çelik M, et al (2007). Analysis of retinal nerve fiber layer thickness in patients with pseudoexfoliation syndrome using optical coherence tomography. Ophthalmologica; 221(5):299-304.

12. Mohamed MM (2009). Detection of early glaucomatous damage in pseudo exfoliation syndrome by assessment of retinal nerve fiber layer thickness. Middle East Afr J Ophthalmol;16(3):141.

13. Ozge G, Koylu MT, Mumcuoglu T, Gundogan FC, Ozgonul C, Ayyildiz O, Kucukevcilioglu M (2016). Evaluation of retinal nerve fiber layer thickness and choroidal thickness in pseudoexfoliative glaucoma and pseudoexfoliative syndrome. Postgraduate Medicine;128(4):444-8.

14. Zangwill LM, Williams J, Berry CC, Knauner S, Weinreb RN (2000). A comparison of optical coherence tomography and retinal nerve fiber layer photography for detection of nerve fibre layer damage in glaucoma. Ophthalmology;107:1309-1315.

15. El Beltagi TA, Bowd C, Boden C, Amini P, Sample PA, Zangwill LM, Weinreb RN (2003). Retinal nerve fiber layer thickness measured with optical coherence tomography is related to visual function in glaucomatous eyes. Ophthalmology;110:2185-2191.

16. Pieroth L, Schuman JS, Hertzmark E, Hee MR, Wilkins JR, et al (1999). Evaluation of focal defects of the nerve fiber layer using optical coherence tomography. Ophthalmology;106:570-579.

17. Harris A, Harris M, Biller J, Garzozi H, Zarfty D, Ciulla TA, Martin B (2000) Aging affects the retrobulbar circulation differently in women and Arch Ophthalmol 118:1076-1080.

18. Lam AK, Chan S-T, Chan H, Chan B (2003) The effect of age on ocular blood supply determined by pulsatile ocular blood flow and color Doppler ultrasonography. Optom Vis Sci 80:305-311.

19. Wiacek MP, Modrzejewska M, Zaborski D (2020). Age-related changes in retrobulbar circulation: a literature review. Int Ophthalmol; 40(2):493-501.

20. Mitchell P, Wang JJ, Smith W (1997) Association of exfoliation syndrome with increased vascular risk. Am J Ophthalmol 124:685-687

21. Naumann GOH, Schlötzer-Schrehardt U, Küchle M. Pseudo exfoliation syndrome for the comprehensive ophthalmologist. Intraocular and systemic manifestations. Ophthalmology 1998;105:951-68.

22. Schlötzer-Schrehardt U, Koca MR, Naumann GOH, Volkholz H (1997). Pseudoexfoliation syndrome. Ocular manifestation of a systemic disorder? Arch Ophthalmol;110:1752-6. 
23. Gottanka J, Flügel-Koch C, Martus P, et al (1997). Correlation of pseudoexfoliative material and optic nerve damage in pseudoexfoliation syndrome. Invest Ophthalmol Vis Sci.;38 (12):2435-2446.

24. Puska P, Vesti E, Tomita G, et al (1999). Optic disc changes in normo tensive persons with unilateral exfoliation syndrome: a 3-year follow-up study. Graefe's Arch Clin Exp Ophthalmol;237(6):457-462.

25. Puska P, Vesti E, Tomita g, Ishida K, Raitta C (1999). Optic disc change in normotensive persons with unilateral exfoliation syndrome: a 3-year follow-up study. Graefe's Arch Clin Exp Ophthalmol;237(6): 457-62.

\section{Tables}

Table 1: Demographic features

\begin{tabular}{|c|c|c|c|c|c|}
\hline & & $\begin{array}{l}\text { Total }(n= \\
95)\end{array}$ & $\begin{array}{l}{ }^{1} \text { PEX (+) Glaucoma }(-)(n \\
=37)\end{array}$ & $\begin{array}{l}\text { 2PEX (+) Glaucoma (+) (n } \\
=14)\end{array}$ & $\begin{array}{l}{ }^{3} \text { Control } \\
(n=44)\end{array}$ \\
\hline $\begin{array}{l}\text { Age } \\
\text { (year) }\end{array}$ & Mean $\pm S s$ & $70,54 \pm 8,93$ & $73,81 \pm 7,46$ & $76,57 \pm 9,21$ & $65,86 \pm 7,65$ \\
\hline \multirow{2}{*}{$\begin{array}{l}\text { Sex; } n \\
(\%)\end{array}$} & Female & $53(55,8)$ & $22(59,5)$ & $6(42,9)$ & $25(56,8)$ \\
\hline & Male & $42(44,2)$ & $15(40,5)$ & $8(57,1)$ & $19(43,2)$ \\
\hline
\end{tabular}

Table 2: Total RNFL, PSV, EDV and RI measurements

\begin{tabular}{|c|c|c|c|c|c|c|c|c|}
\hline & \multirow{2}{*}{$\begin{array}{l}\text { 1PEX (+) } \\
\text { Glaucoma }(-)(n \\
=53) \\
\text { Mean } \pm \text { Sd }\end{array}$} & \multirow{2}{*}{$\begin{array}{l}\text { 2PEX }(+) \\
\text { Glaucoma }(+)(n \\
=18) \\
\text { Mean } \pm \text { Sd }\end{array}$} & \multirow{2}{*}{$\begin{array}{l}{ }^{3} \text { Control } \\
(n=44) \\
\text { Mean } \pm S d\end{array}$} & \multirow[t]{2}{*}{${ }^{a} p$} & \multicolumn{3}{|c|}{ Post Hoc } & \\
\hline & & & & & ${ }^{b} p_{1-2}$ & ${ }^{b} \boldsymbol{p}_{1-3}$ & ${ }^{b} p_{2-3}$ & \\
\hline $\begin{array}{l}\text { Total } \\
\text { RNFL }\end{array}$ & $87,91 \pm 14,97$ & $90,17 \pm 12,74$ & $96,18 \pm 11,90$ & $0.012^{*}$ & 1,000 & $0.010^{*}$ & 0,345 & $\begin{array}{l}3 \\
> \\
1\end{array}$ \\
\hline PSV & $41,26 \pm 15,45$ & $39,54 \pm 15,41$ & $52,94 \pm 15,34$ & $0.001^{* *}$ & 1,000 & $0.001 * *$ & $0.007^{* *}$ & $\begin{array}{l}3 \\
> \\
1, \\
2\end{array}$ \\
\hline EDV & $12,45 \pm 5,71$ & $11,54 \pm 5,68$ & $16,56 \pm 5,19$ & $0.001 * *$ & 1,000 & $0.001^{* *}$ & $0.004^{* *}$ & $\begin{array}{l}3 \\
> \\
1, \\
2\end{array}$ \\
\hline RI & $0,70 \pm 0.06$ & $0,70 \pm 0.07$ & $0,68 \pm 0.06$ & 0,370 & 1,000 & 0,765 & 0,672 & - \\
\hline
\end{tabular}

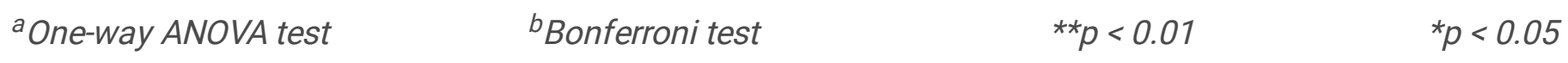

Table 3: Evaluation of RNFL measurements 


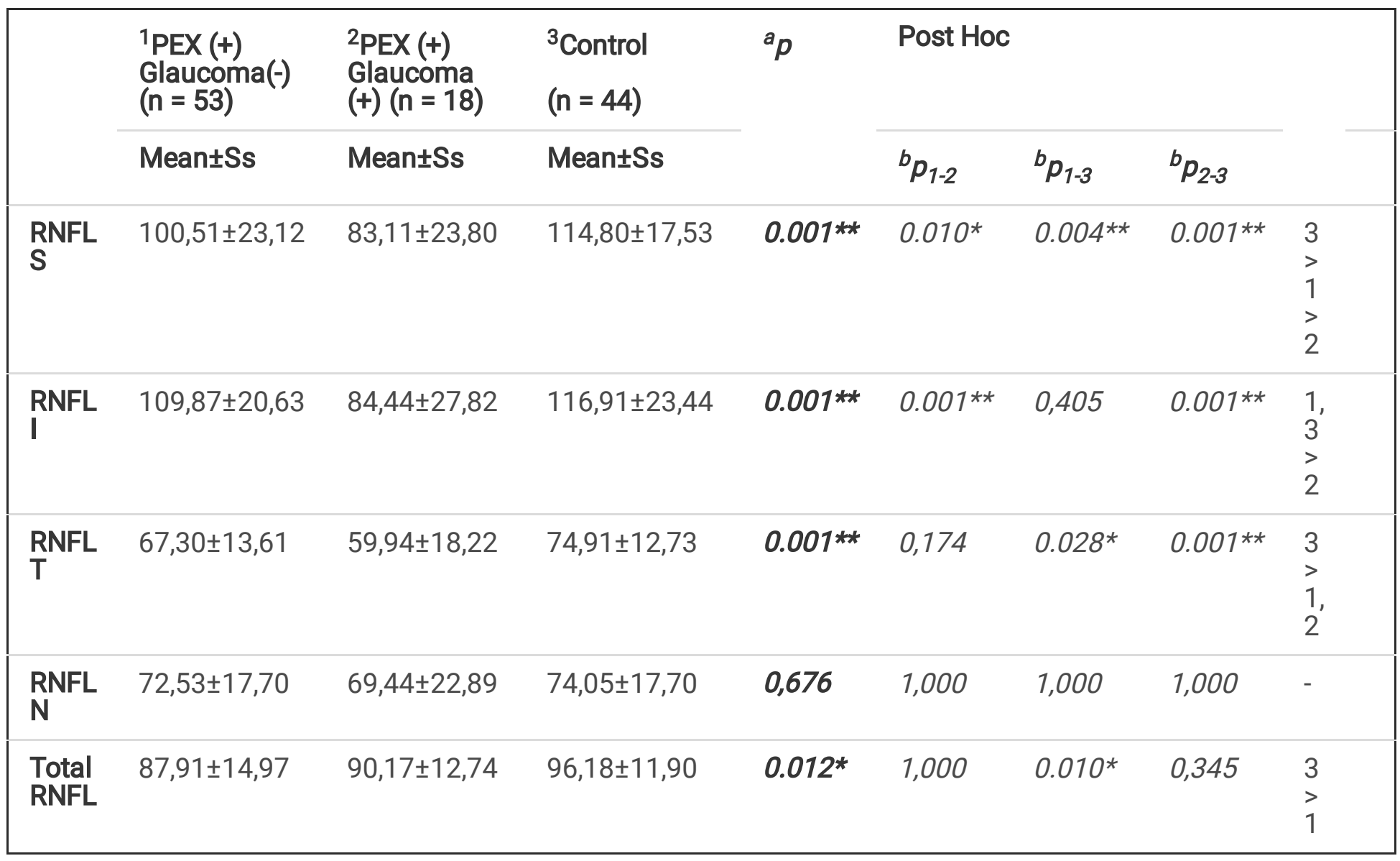

${ }^{a}$ One-way ANOVA test

${ }^{b}$ Bonferroni test

${ }^{* *} p<0.01$

${ }^{*} p<0.05$

Table 4: Relationship between total RNLF and PSV, EDV and RI by group

\begin{tabular}{|c|c|c|c|c|c|c|}
\hline & \multicolumn{6}{|c|}{ Total RNFL } \\
\hline & \multicolumn{2}{|c|}{${ }^{1}$ PEX (+) Glaucoma (-) } & \multicolumn{2}{|c|}{${ }^{2}$ PEX (+) Glaucoma (+) } & \multicolumn{2}{|c|}{${ }^{3}$ Control } \\
\hline & $\mathbf{R}$ & $P$ & $\mathbf{R}$ & $P$ & $r$ & $p$ \\
\hline PSV & $-0,164$ & 0,240 & $-0,743$ & $0.001 * *$ & 0,317 & $0.036^{*}$ \\
\hline EDV & $-0,207$ & 0,138 & $-0,691$ & $0.001 * *$ & 0,337 & $0.025^{*}$ \\
\hline RI & 0.095 & 0,499 & $-0,152$ & 0,548 & -0.046 & 0,766 \\
\hline
\end{tabular}

Pearson correlation analysis

${ }^{*} p<0.05$

$* * p<0.01$

\section{Figures}




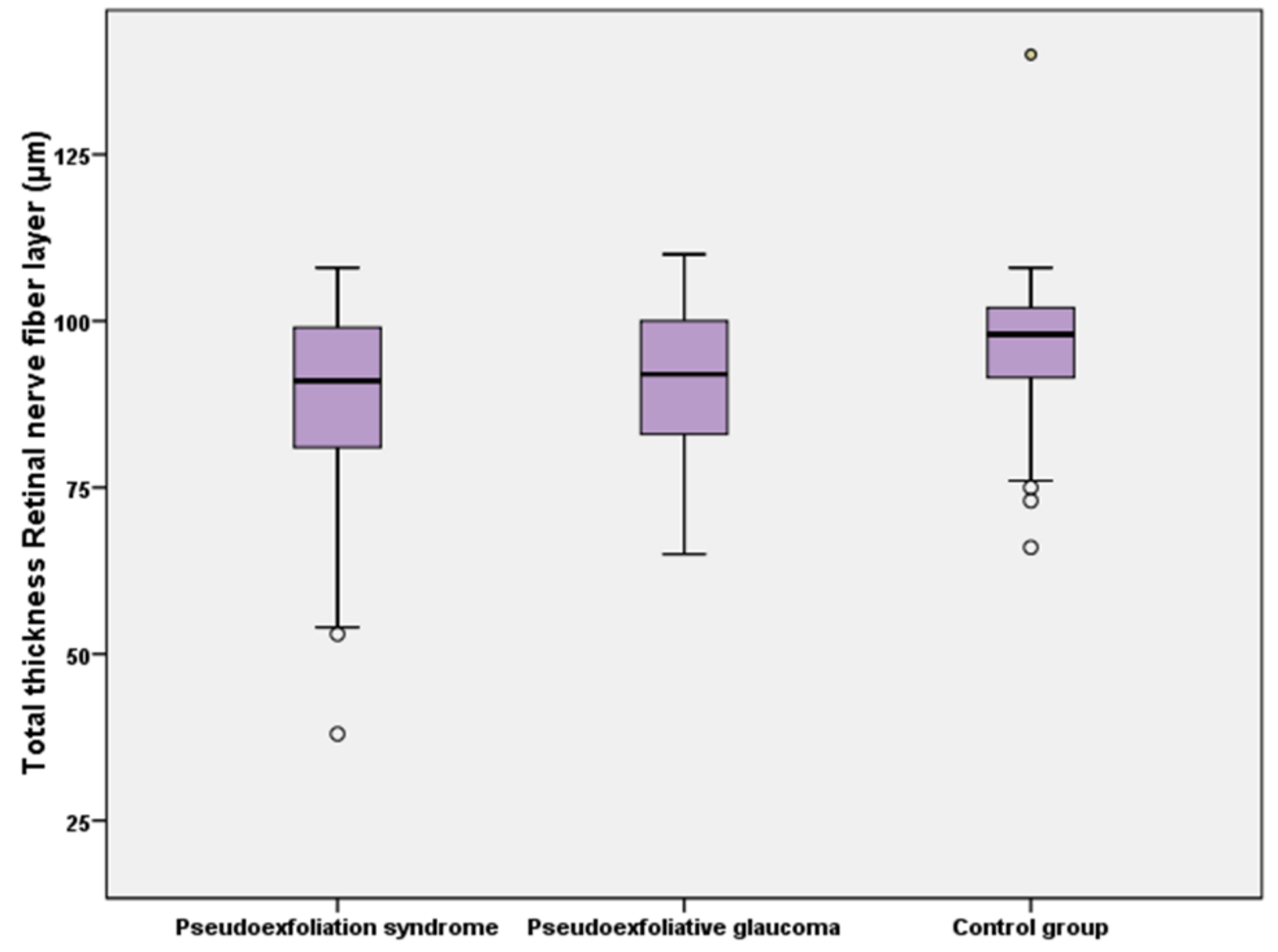

Figure 1

Total RNFL thickness distribution by group 


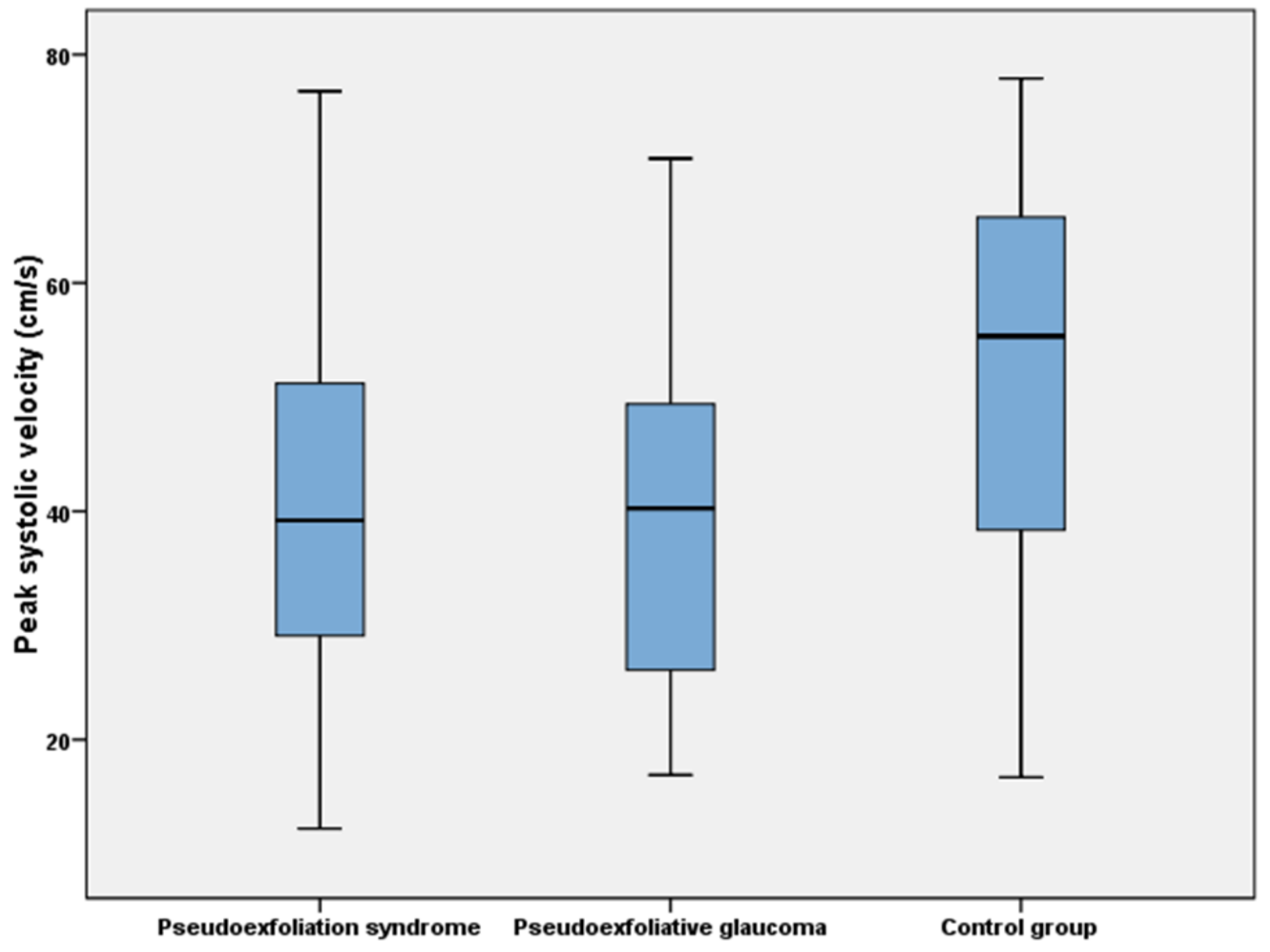

Figure 2

PSV distribution by group 


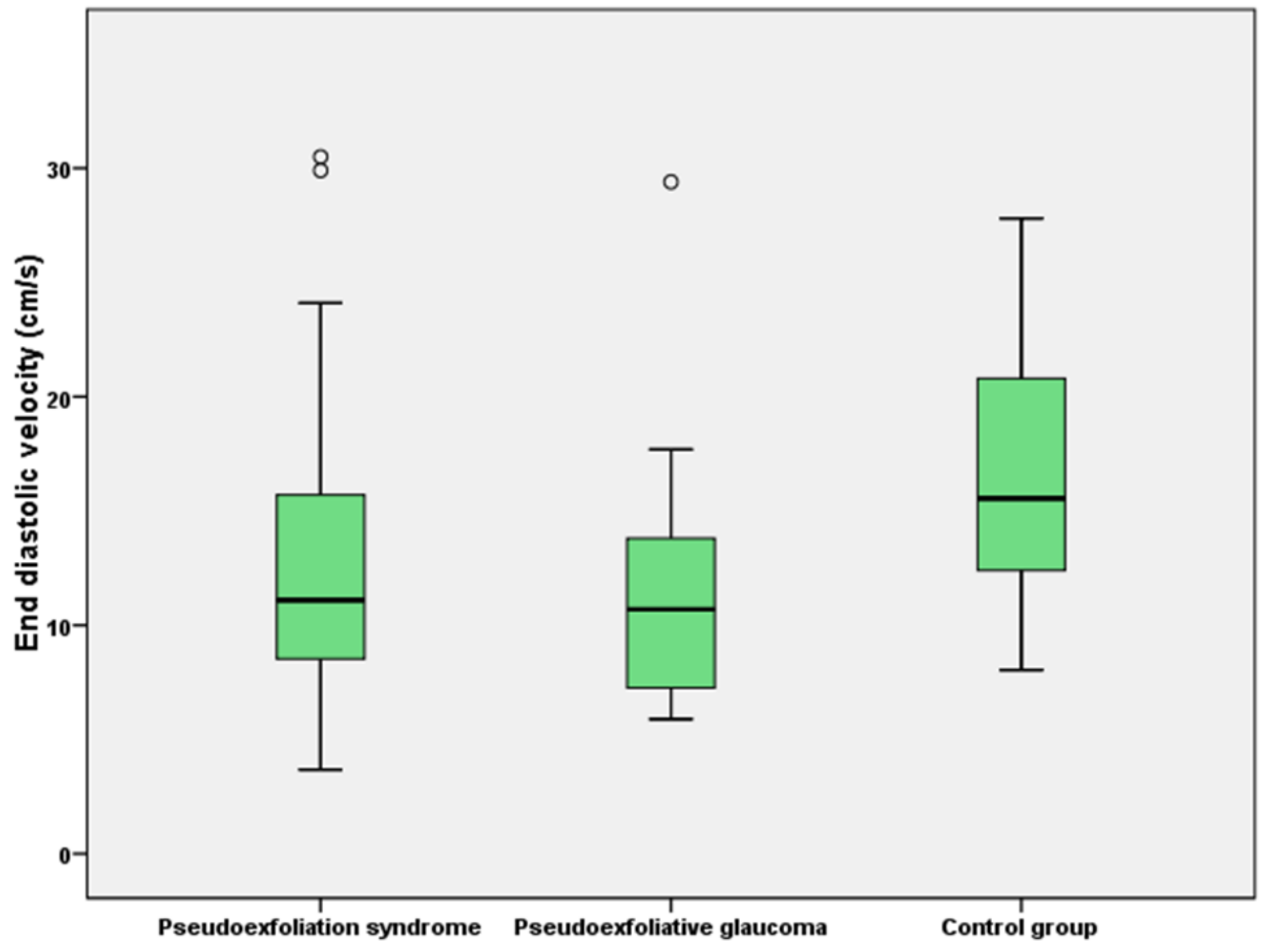

Figure 3

EDV distribution by group 


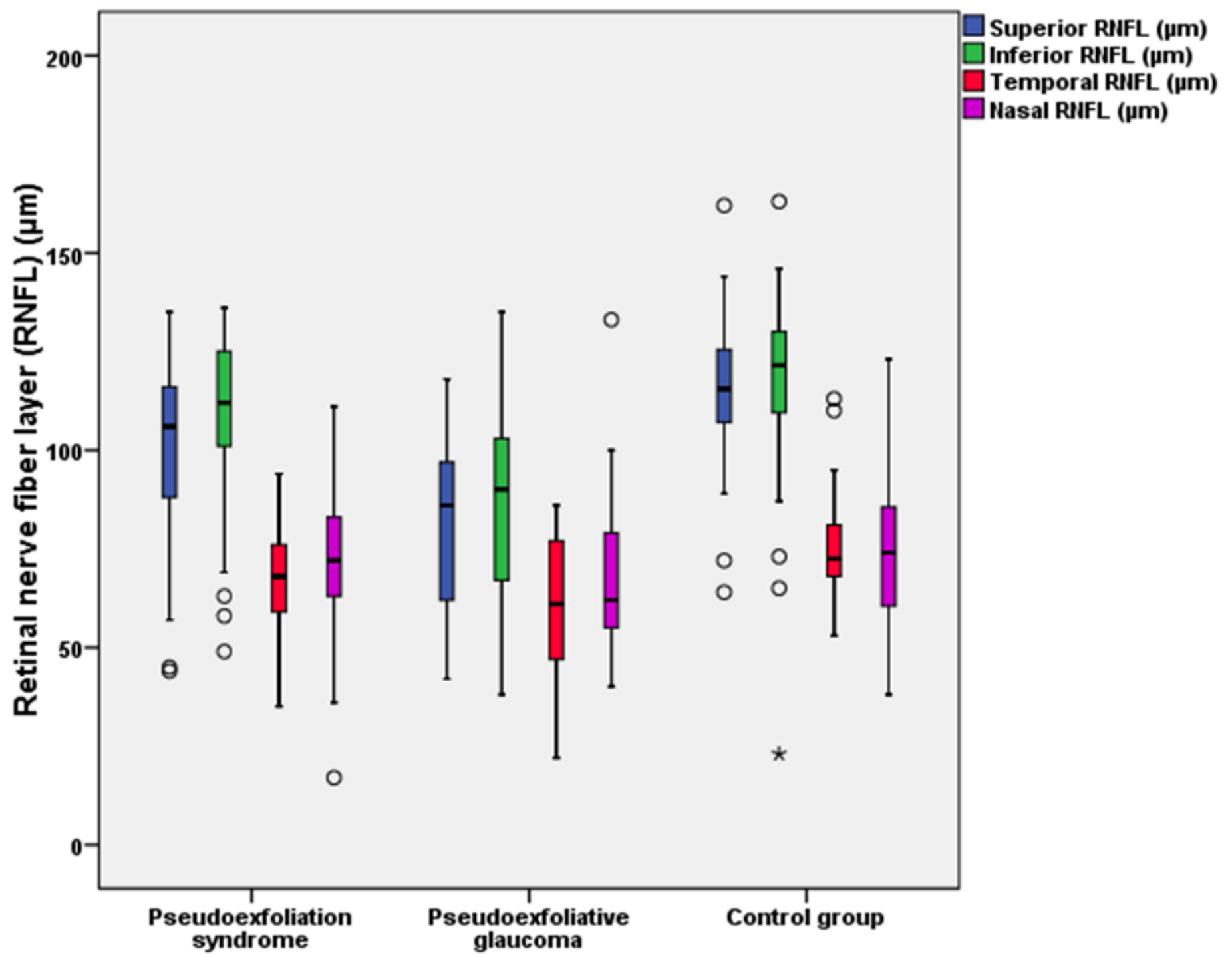

Figure 4

RNFL quadrant distribution by group 


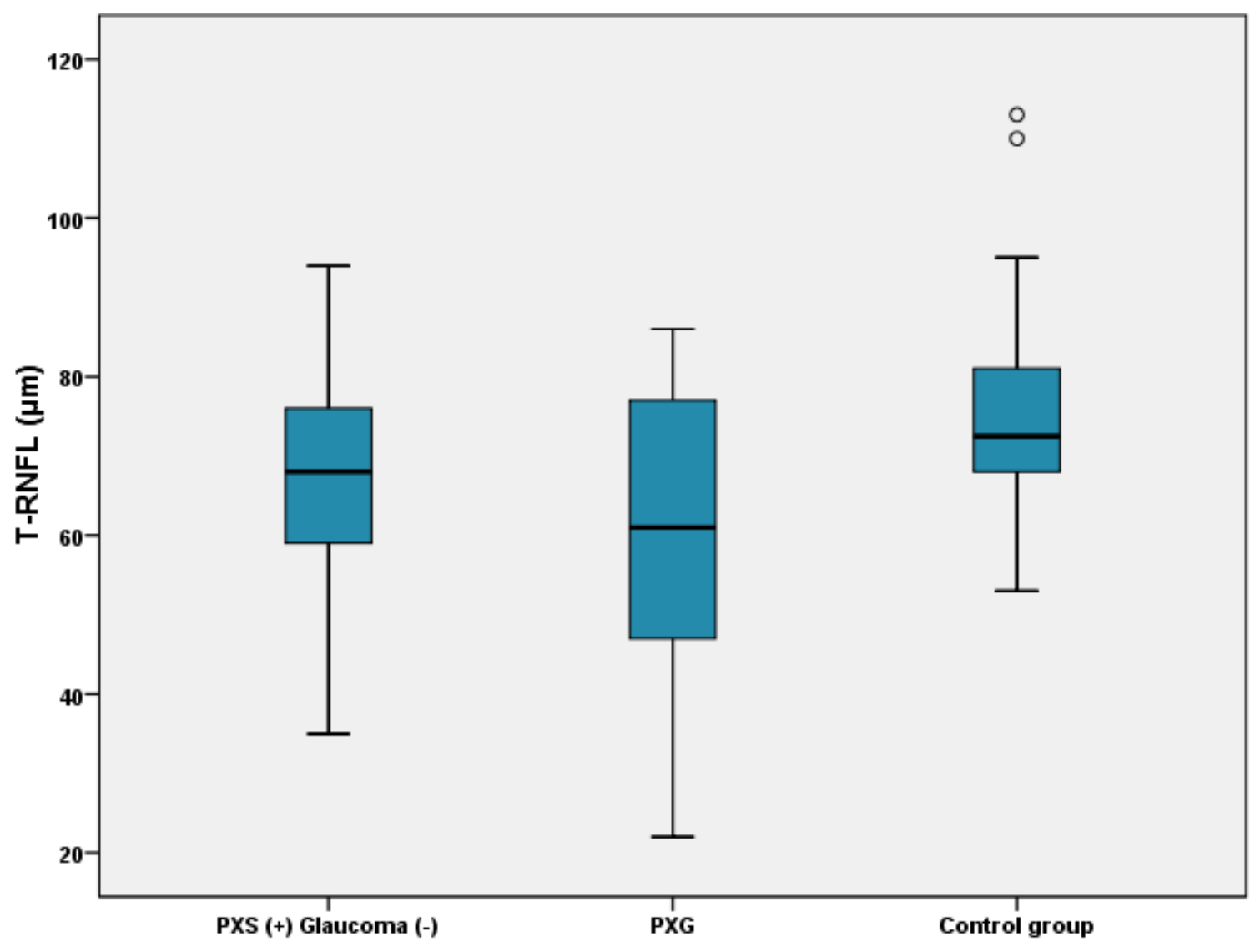

Figure 5

RNFL T quadrant distribution by group T-RNFL: Temporal retinal nerve fiber layer, PXS: Pseudoexfoliation syndrome, PXG: Pseudoexfoliative glaucoma 